\title{
Investigation of combining ability and superiority percentages for yield and some related traits in yellow maize using line $\times$ tester analysis
}

\author{
Maamoun A. Abdel-Moneam ${ }^{1}$ - M. S. Sultan ${ }^{1}$ - Waleed A. E. Abido ${ }^{1}$ - Ágnes Hadházy ${ }^{2}$ - S. A. Sadek ${ }^{3}$ - \\ M. S. Shalof ${ }^{3}$ \\ ${ }^{1}$ Agronomy Department, Faculty of Agriculture, Mansoura University, Egypt, \\ ${ }^{2}$ Research Institute of Nyiregyhaza, Institutes for Agricultural Research and Educational Farm, University of Debrecen, Hungary \\ ${ }^{3}$ Maize Research Department, Field Crop Institute, Agriculture Research Center, Egypt \\ madawy78@mans.edu.eg
}

\begin{abstract}
SUMMARY
Combining ability estimation is an important genetic attribute for maize breeders in anticipating improvement in productivity via hybridization and selection. This research was carried out to investigate the genetic structure of the $27 F_{1}$ maize hybrids established from nine lines derived from Maize Research Department and three testers, to determine general combining ability (GCA), determine crosses showing specific combining ability (SCA) and superiority percentages for crosses. Nine lines, three testers, $27 F_{1}$ hybrids and two check commercial hybrids (SC162 and SC168) were studied in randomized complete block Design (RCBD) with three replications during 2016. The results of mean squares showed that significant and highly significant for most studied traits (days to 50\% tasseling, days to 50\% silking, plant and ear height, ear position, ear length, no. of kernels per row, 100-kernel weight and Grain yield). Estimates of variance due to GCA and SCA and their ratio revealed predominantly non-additive gene effects for all studied traits. Lines with the best GCA effects were: $P_{2}$ (line 11) and $P_{6}$ (line 21$)$ for grain yield, for testers Gm174 and Gm1021 had significant GCA effects for grain yield. The hybrids $P_{5} \times G m 1021, P_{6} \times G m 1021, P_{7} \times G m 1021$, $P_{8} \times G m 1002, P_{9} \times G m 1002$ had significant and negative SCA effects for grain yield. Crosses $P_{1} \times G m 174, P_{2} \times G m 1002, P_{5} \times G m 1021$, $P_{6} \times G m 174, P_{6} \times G m 1021, P_{7} \times G m 1021, P_{8} \times G m 1002, P_{9} \times G m 1021$ were the best combinations manifested and significant superiority percentages over than check varieties (SC162 and SC168) for most studied traits. Therefore, these hybrids may be preferred for hybrid crop development.

Abbreviations: GCA general combining ability; SCA specific combining ability
\end{abstract}

Keywords: Maize, line $\times$ tester, general combining ability, specific combining ability

\section{INTRODUCTION}

Maize crop is extensively grown as grain for humans and fodder for livestock consumption. Maize is one of the most important grain crops in Egypt; Total area under cultivation of maize in Egypt 1082766 ha of the total cultivated agricultural land, while average yield is 8.40 ton ha $^{-1}$ (FAOSTAT, 2016). Estimation of combining ability and genetic variance components are important in the breeding programs for hybridization. In any breeding program, the choice of the correct Parents is the secret of the success. One of the most important criteria in breeding programs for identifying the hybrids with high yield is knowledge of parent genetic structure and information regarding their combining ability (Ceyhan et al., 2008). For maize yield, they observed that the importance of general combining ability was relatively more than specific combining ability for unselected inbred lines, while specific combining ability was more important than general combining ability for previously selected lines. General combining ability is a good estimate of additive gene action, whereas specific combining ability is a measure of non-additive gene action (Sharief et al., 2009). The study of Line $\times$ tester analysis of the genetic traits would certainly be a valuable aid in selection and breeding for better maize hybrids. Line $\times$ tester mating design was developed by Kempthorne (1957), which provides reliable information on the general and specific combining ability effects of parents and their hybrid combinations in applied breeding programs (Sharma et al., 2004). Rahman et al., (2010) tested 24 maize $S^{2}$ lines using line $x$ tester analysis for some traits related to earlier flowering and ear height and revealed highly significant differences among the testcrosses for measured parameters. Asefa et al. (2008) conducted an experiment to determine the combining ability of highland maize inbred lines for ear length, 1000-kernel weight, ear height, shelling percentage and grain yield. The present study was aimed to evaluating the general combining ability and specific combining ability for grain yield and some related traits and to identify and select the superior hybrid combinations based on crosses of selected lines with testers.

\section{MATERIALS AND METHODS}

The nine lines and three testers in (Table 1) were crossed in $9 \times 3$ (line $\times$ tester) to produce 27 possible $F_{1}$ hybrids. Nine lines, three testers, $\mathrm{F}_{1}$ hybrids and the two commercial check hybrids i.e. SC162, SC168 were grown at the Agricultural Research Center in Egypt during 2016 summer season in two locations (Gemmeiza and Mallawy). The experiment was a randomized complete block design (RCBD) with three replications. The genotypes were assigned at random to experimental unit in each block and each row contained 25 plants. Each replication consisted of 12 parents (lines and tester), $27 \mathrm{~F}_{1}$ crosses and two check hybrids with a 5 meter long and one row for each genotype. Inter-plant and inter-row distances were as 20 and 70 
$\mathrm{cm}$, respectively (Plot size was: $5 \mathrm{~m} \times 70 \mathrm{~cm}=3.5$ $\mathrm{m}^{2} /$ plot, No. of rows in fadden $=4200 / 3.5=1200$ rows /fad. and number of plants in fadden $=1200 \times 25=30000$ plants/fad). Weeds were removed manually, when necessary. The research traits including days to $50 \%$ tasseling (TS), days to 50\% silking (SK), plant and ear heights (PH, EH), ear position (EP), late wilt disease (LW), ear length (EL), ear diameter (ED), No. of rows per ear (RN), no. of kernels per row (KN), 100-kernel weight $(\mathrm{KW})$ and grain yield per faddan (GY) were evaluated based on standard evaluation system maize. The breeding value of the plant material was evaluated by analyzing the combining ability for all the traits in their combined data. Data were recorded on 5 plants are taken randomly selected plant samples.

Names and the pedigree of the studied twelve yellow inbred lines

\begin{tabular}{|c|c|c|c|}
\hline No. & Inbred line & Pedigree & Notes \\
\hline 1 & line 10 & EG-38-B5-2-77-1-1-1 & Line \\
\hline 2 & line 11 & EG-29-B5-2-57-2-1-1 & Line \\
\hline 3 & line 12 & Gm.Y.Pop.F14 & Line \\
\hline 4 & line 17 & EG-28-B5-2-131-2-3-1 & Line \\
\hline 5 & line 20 & EG-28-B5-2-127-1-1-1 & Line \\
\hline 6 & line 21 & Gm. y. Pop. F 21 & Line \\
\hline 7 & line 26 & EG-29-B5-2-186-1-1-1 & Line \\
\hline 8 & line 32 & Sc.2-F47-48/A2- 2003 & Line \\
\hline 9 & line 48 & EG-26-B5-1-49-1-1-1 & Line \\
\hline 10 & line Gm. 174 & EG-40-B5-2-104-2-1-1 & Tester \\
\hline 11 & line Gm.1002 & Sub trop. _ Y. I.G. S. Pop._IITA_N.M.B.P. & Tester \\
\hline 12 & line Gm.1021 & IL. Sd - $121 \times$ Pop. (DMR- ESR) & Tester \\
\hline
\end{tabular}

Gm.1002 and Gm.1021 were developed at Gemmeiza Agricultural Research Station during the period of 1983 to 1992 by S. E. Sadek et al., NMBP, FCRI, ARC, Egypt

\section{Statistical analysis}

Combining ability studies were determined by using line $x$ tester analysis as described by
Kempthrone (1957). Data were analyzed using the following statistical model: For combined analyses

$$
\mathrm{X}_{\mathrm{lijk}}=\mu+\mathrm{L}_{1}+\mathrm{Rs} / \mathrm{L}_{1}+\mathrm{g}_{\mathrm{i}}+\mathrm{g}_{\mathrm{j}}+\mathrm{S}_{\mathrm{ij}}+\left(\mathrm{Lg}_{\mathrm{i}}\right)_{\mathrm{li}}+\left(\mathrm{Lg}_{\mathrm{j}}\right)_{\mathrm{lj}}+\left(\mathrm{LS}_{\mathrm{ij}}\right)_{\mathrm{ijj}}+\ell_{\mathrm{isij}}
$$

$\mu=$ over all genotype mean; $\mathrm{L}_{\mathrm{i}}=$ locations effects; $\mathrm{Rs} / \mathrm{L}_{\mathrm{i}}$ $=$ replications within locations effects; $g_{i}=$ G.C.A. effect of the $i$ the male parents (testers); $g_{j}=$ G.C.A. effect of the $\mathrm{j}$ the female parents (inbred line); $\mathrm{S}_{\mathrm{ij}}=$ S.C.A. effect of the ij the cross combinations; $\left(\operatorname{Lg}_{\mathrm{i}}\right)_{\mathrm{li}}=$ interaction of location $x$ males (testers) effects; $\left(\operatorname{Lg}_{\mathrm{j}}\right)_{\mathrm{lj}}=$ interaction of location $\mathrm{x}$ female (inbred lines) effects; $\left(\mathrm{LS}_{\mathrm{ij}}\right)_{\mathrm{ilj}}=$ interaction of between location, males and female effects; $\ell_{\text {isij }}=$ the error associated with the each observation; Then, data were analyzed using Agrobase 21 (2001) and Microsoft excel. Analysis of traits from the lines, testers and crosses were conducted using the line by tester (AGR 21) procedure developed, according to Method line by tester, which included the parents, direct and crosses. The LSD test at 5\% and $1 \%$ according to Steel and Torrie (1980) was used for comparison the mean of performance of the different genotypes.

\section{RESULTS AND DISCUSSION}

\section{Analysis of variance}

For mean squares in Table $(2,3)$ showed that significant and highly significant for most studied traits (days to 50\% tasseling, days to 50\% silking, plant and ear height, ear position, ear length, no. of kernels per row, 100-kernel weight and grain yield). For late wilt disease showed highly significant in location, rep., Par. vs.crosses and testers. For ear diameter showed highly significant in Parents vs.crosses. For No. of rows per ear significant and highly significant in location, rep., genotypes and Parents vs.crosses. These results agree with those obtained by Sultan, et al., 2010; Atif et al., 2012; Moosavi et al., 2012 and Kamara, et al., 2014. 
Analysis of variance for days to $50 \%$ tasseling, days to $50 \%$ silking, plant and ear height, ear position and late wilt disease in their combined data over locations

\begin{tabular}{|c|c|c|c|c|c|c|c|}
\hline \multirow{2}{*}{ SOV } & \multirow{2}{*}{ df } & \multicolumn{6}{|c|}{ Combined location } \\
\hline & & TS & SK & $\mathrm{PH}$ & EH & EP & LW \\
\hline Locations & 1 & $9.20 * *$ & $33.85^{* *}$ & $12701.6^{* *}$ & $7244.46^{* *}$ & $156.97 * *$ & $31.61 * *$ \\
\hline Rep. & 5 & $61.60 * *$ & $15.73 * *$ & $2548.97 * *$ & $1475.82 * *$ & $38.10^{* *}$ & $7.81 * *$ \\
\hline Rep. $\times$ Location & 4 & $9.15^{* *}$ & $11.20 * *$ & $10.80^{* *}$ & $33.66^{* *}$ & $8.41^{*}$ & 1.86 \\
\hline Genotypes & 38 & $22.64 * *$ & $22.96 * *$ & $3719.08 * *$ & $1067.77 * *$ & $18.79 * *$ & 2.05 \\
\hline Parents & 11 & $19.10^{* *}$ & $19.97 * *$ & $581.41 * *$ & $229.24 * *$ & $39.78^{* *}$ & 0.23 \\
\hline Crosses & 26 & $7.06^{* *}$ & $7.13 * *$ & $212.11 * *$ & $114.13^{* *}$ & $10.18^{* *}$ & 2.46 \\
\hline Par.vs.crosses & 1 & $466.80^{* *}$ & $467.27 * *$ & $129414.6^{* *}$ & $35086.10^{* *}$ & $11.43^{* *}$ & $11.41^{* *}$ \\
\hline Lines & 8 & $4.72 *$ & $3.92 *$ & $171.07 * *$ & $113.06^{* *}$ & $5.88 *$ & 1.99 \\
\hline Testers & 2 & $24.13 * *$ & $24.03 * *$ & $238.35^{* *}$ & $184.47 * *$ & $9.62 * *$ & $7.21 * *$ \\
\hline Lines $\times$ testers & 16 & $6.09 * *$ & $6.62^{* *}$ & $229.35^{* *}$ & $105.87 * *$ & $12.41 * *$ & 2.11 \\
\hline Error & 152 & 2.87 & 2.69 & 84.35 & 49.64 & 9.29 & 0.69 \\
\hline
\end{tabular}

$*$, ** significance at 0.05 and 0.01 level of probability, respectively.

Table 3

Analysis of variance for ear length, ear diameter, no. of rows per ear, no. of kernels per row, 100-kernel weight and grain yield in their combined data over location

\begin{tabular}{|c|c|c|c|c|c|c|c|}
\hline \multirow{2}{*}{ SOV } & \multirow{2}{*}{ df } & \multicolumn{6}{|c|}{ Combined location } \\
\hline & & EL & ED & $\mathrm{RN}$ & $\mathrm{KN}$ & KW & GY \\
\hline Location & 1 & $20.70 * *$ & 0.19 & $117.34 * *$ & $8.05^{*}$ & $28.81 * *$ & $110.83 * *$ \\
\hline Rep. & 5 & $6.21 *$ & 0.31 & $25.65 * *$ & $14.53 * *$ & $73.18 * *$ & $24.72 * *$ \\
\hline Rep.$\times$ Location & 4 & 2.69 & 0.22 & 1.28 & $23.35^{* *}$ & $84.52 * *$ & 3.20 \\
\hline Genotypes & 38 & $47.14^{* *}$ & 1.08 & $7.03 * *$ & $215.77 * *$ & $169.48^{* *}$ & $689.47 * *$ \\
\hline Parents & 11 & $10.62 * *$ & 0.27 & $6.79 *$ & $65.17 * *$ & $25.77 * *$ & $46.94 * *$ \\
\hline Crosses & 26 & $16.59 * *$ & 0.27 & 2.64 & $25.32 * *$ & $46.97 * *$ & $66.09 * *$ \\
\hline Par. vs.crosses & 1 & $1243.08 * *$ & $30.88 * *$ & $123.75^{* *}$ & $6824.16^{* *}$ & $4935.51 * *$ & $23965.5 * *$ \\
\hline Lines & 8 & $7.53 *$ & 0.22 & 3.59 & $20.27 * *$ & $40.85 * *$ & $56.04 * *$ \\
\hline Testers & 2 & $46.23 * *$ & 0.69 & 1.75 & $40.49 * *$ & $163.78^{* *}$ & $165.89 * *$ \\
\hline Lines $\times$ testers & 16 & $17.42 * *$ & 0.25 & 2.28 & $25.95 * *$ & $35.42 * *$ & $58.63 * *$ \\
\hline Error & 152 & 3.57 & 0.18 & 1.07 & 20.06 & 16.84 & 7.38 \\
\hline
\end{tabular}

*, ** significance at 0.05 and 0.01 level of probability, respectively.

\section{General combining ability effects}

Results of GCA effects (Table 4 and 5) for days to $50 \%$ tasseling for lines showed negative and significant GCA effect for $\mathrm{P}_{4}$ (line 17) and $\mathrm{P}_{9}$ (line 48) in plant height, $\mathrm{P}_{6}$ (line 21) in ear height and $\mathrm{P}_{7}$ (line 26) in late wilt disease. Testers showed significant and negative GCA effect for Gm174 in late wilt disease and Gm1021 in days to $50 \%$ tasseling date, days to $50 \%$ silking date and ear height. While, positive and significant GCA effects for line $\mathrm{P}_{6}$ (line 21) in ear length, ear diameter, No. or rows per ear and grain yield and $\mathrm{P}_{2}$ (line 11) and $\mathrm{P}_{2}$ (line 32) in grain yield. For testers, Gm174 in ear length, and grain yield and Gm1021 in ear length, 100kernel weight and grain yield. These results are in conformity by the finding of Welcker et al. (2005), Rakesh et al. (2006), Osman and Ibrahim (2007), Singh and Roy (2007), Parmar (2007), EL-Shenawy et al. (2009) and Sultan et al. (2010) and Atif et al. (2012). 
Estimates of GCA effects of nine parents and three testers of yellow maize inbreds for days to $50 \%$ tasseling, days to $50 \%$ silking, plant and ear height, ear position and late wilt disease in their combined over locations

\begin{tabular}{|c|c|c|c|c|c|c|c|}
\hline & & \multicolumn{6}{|c|}{ Combined locations } \\
\hline & & TS & SK & PH & $\mathrm{EH}$ & EP & LW \\
\hline$P_{1}($ line 10$)$ & & $1.13 * *$ & $0.95^{*}$ & 3.96 & 2.58 & 0.23 & 0.22 \\
\hline $\mathrm{P}_{2}$ (line 11) & & -0.37 & -0.33 & 1.24 & -0.09 & -0.31 & -0.34 \\
\hline $\mathrm{P}_{3}$ (line 12) & & 0.13 & 0.12 & 1.52 & 2.36 & 0.70 & 0.27 \\
\hline $\mathrm{P}_{4}$ (line 17) & & 0.41 & 0.34 & $-4.26^{*}$ & -2.64 & -0.15 & -0.12 \\
\hline $\mathrm{P}_{5}$ (line 20) & & -0.09 & -0.22 & 2.41 & 2.47 & 0.55 & 0.55 \\
\hline $\mathrm{P}_{6}($ line 21$)$ & & -0.09 & 0.12 & -2.43 & $-3.48 *$ & -1.04 & 0.27 \\
\hline $\mathrm{P}_{7}$ (line 26) & & -0.26 & 0.01 & 2.52 & 2.41 & 0.57 & $-0.40^{*}$ \\
\hline $\mathrm{P}_{8}$ (line 32) & & -0.26 & -0.33 & -0.48 & -1.31 & -0.48 & -0.23 \\
\hline $\mathrm{P}_{9}$ (line 48) & & -0.59 & -0.66 & $-4.48^{*}$ & -2.31 & -0.07 & -0.23 \\
\hline \multirow{2}{*}{ LSD } & 0.05 & 0.76 & 0.74 & 4.23 & 3.25 & 1.39 & 0.37 \\
\hline & 0.01 & 1.00 & 0.97 & 5.56 & 4.27 & 1.82 & 0.48 \\
\hline Gm 174 & & -0.26 & -0.31 & 0.94 & 1.43 & 0.44 & $-0.25^{*}$ \\
\hline Gm 1002 & & $0.76^{* *}$ & $0.77 * *$ & 1.46 & 0.65 & -0.05 & $0.42^{* *}$ \\
\hline Gm 1021 & & $-0.50 *$ & $-0.46^{*}$ & -2.41 & $-2.09 *$ & -0.40 & -0.17 \\
\hline \multirow{2}{*}{ LSD } & 0.05 & 0.45 & 0.43 & 2.43 & 1.86 & 0.80 & 0.21 \\
\hline & 0.01 & 0.59 & 0.56 & 3.19 & 2.44 & 1.05 & 0.28 \\
\hline
\end{tabular}

$*, * *$ significance at 0.05 and 0.01 level of probability, respectively

Table 5

Estimates of GCA effects of nine parents and three testers of yellow maize inbreds for ear length, ear diameter, no. of rows per ear, no. of kernels per row, 100-kernel weight and grain yield in their combined over locations

\begin{tabular}{|c|c|c|c|c|c|c|c|}
\hline & & \multicolumn{6}{|c|}{ Combined locations } \\
\hline & & EL & ED & $\mathrm{RN}$ & $\mathrm{KN}$ & $\mathrm{KW}$ & GY \\
\hline $\mathrm{P}_{1}$ (line 10) & & -0.16 & -0.04 & -0.12 & -1.23 & -1.84 & 0.53 \\
\hline $\mathrm{P}_{2}$ (line 11) & & 0.73 & 0.02 & 0.05 & 0.10 & 1.72 & $1.85^{* *}$ \\
\hline $\mathrm{P}_{3}$ (line 12) & & -0.83 & 0.07 & -0.67 & 0.77 & -2.62 & -1.18 \\
\hline $\mathrm{P}_{4}$ (line 17) & & -0.72 & -0.15 & -0.40 & -1.17 & -0.45 & -0.78 \\
\hline $\mathrm{P}_{5}$ (line 20) & & -0.22 & -0.04 & 0.38 & -0.12 & 0.99 & -3.31 \\
\hline $\mathrm{P}_{6}($ line 21$)$ & & $1.17 * *$ & $0.24 *$ & $0.83^{* *}$ & 0.38 & 0.60 & $2.17 * *$ \\
\hline $\mathrm{P}_{7}$ (line 26) & & 0.23 & -0.04 & -0.12 & 0.99 & -0.28 & -0.74 \\
\hline $\mathrm{P}_{8}$ (line 32) & & 0.12 & 0.02 & 0.27 & 1.60 & 1.83 & $1.70^{* *}$ \\
\hline $\mathrm{P}_{9}$ (line 48) & & -0.33 & -0.09 & -0.23 & -1.34 & 0.05 & -0.24 \\
\hline \multirow{2}{*}{ LSD } & 0.05 & 0.86 & 0.19 & 0.47 & 2.05 & 1.88 & 1.25 \\
\hline & 0.01 & 1.13 & 0.25 & 0.61 & 2.70 & 2.47 & 1.64 \\
\hline Gm 174 & & $0.51 *$ & 0.07 & -0.15 & 0.70 & 0.88 & $1.00 * *$ \\
\hline Gm 1002 & & -1.07 & -0.13 & -0.04 & -0.97 & -2.01 & -2.02 \\
\hline Gm 1021 & & $0.56^{*}$ & 0.06 & 0.20 & 0.27 & 1.13 & $1.02^{* *}$ \\
\hline \multirow{2}{*}{ LSD } & 0.05 & 0.49 & 0.113 & 0.27 & 1.17 & 1.07 & 0.70 \\
\hline & 0.01 & 0.64 & 0.149 & 0.36 & 1.54 & 1.41 & 0.92 \\
\hline
\end{tabular}

$*, * *$ significance at 0.05 and 0.01 level of probability, respectively 


\section{Specific combining ability effects}

Results in Tables $(6,7)$ for SCA effect showed that $\mathrm{P}_{1} \times \mathrm{Gm} 174$ had significant and negative SCA effects for days to $50 \%$ tasseling date and positive significantly SCA effects in ear length, 100-kernel weight and grain yield. $\mathrm{P}_{1} \times \mathrm{Gm} 1021$ had significant and negative SCA effects for plant height. $\mathrm{P}_{2} \times \mathrm{Gm} 1002$ had significant and positive SCA effects for ear length and grain yield. $\mathrm{P}_{3} \times \mathrm{Gm} 1002$ had significant and negative SCA effects for resistance to late wilt disease and positive SCA effects for ear length. $\mathrm{P}_{4} \times \mathrm{Gm} 174$ had significant and positive SCA effect for ear length. $\mathrm{P}_{4} \times \mathrm{Gm} 11021$ had significant and positive SCA effects for 100 -kernel weight. $\mathrm{P}_{5} \times \mathrm{Gm} 1002$ had significant and positive SCA effect for no. of rows per ear. $\mathrm{P}_{5} \times \mathrm{Gm} 1021$ had significant and positive SCA effects for ear length and grain yield. $\mathrm{P}_{6} \times \mathrm{Gm} 1021$ had significant and negative SCA effects for resistance to late wilt disease and positive SCA effect for grain yield. $\mathrm{P}_{7} \times \mathrm{Gm} 1021$ had significant and positive SCA effect for ear length and grain yield. $\mathrm{P}_{8} \times \mathrm{Gm} 1002$ had significant and negative SCA effects for ear height and ear position. $\mathrm{P}_{9} \times \mathrm{Gm} 1002$ had significant and positive SCA effects for grain yield. These results are in line with those obtained by Osman and Ibrahim (2007), Singh and Roy (2007), Parmar (2007), Liu and Tollenaar (2009), Fan et al. (2009) and Atif et al. (2012).

Estimates of SCA effects of 27 yellow single crosses of maize at their combined data over locations for days to $50 \%$ tasseling, days to $50 \%$ silking, plant and ear height, ear position and late wilt disease in growing season 2016

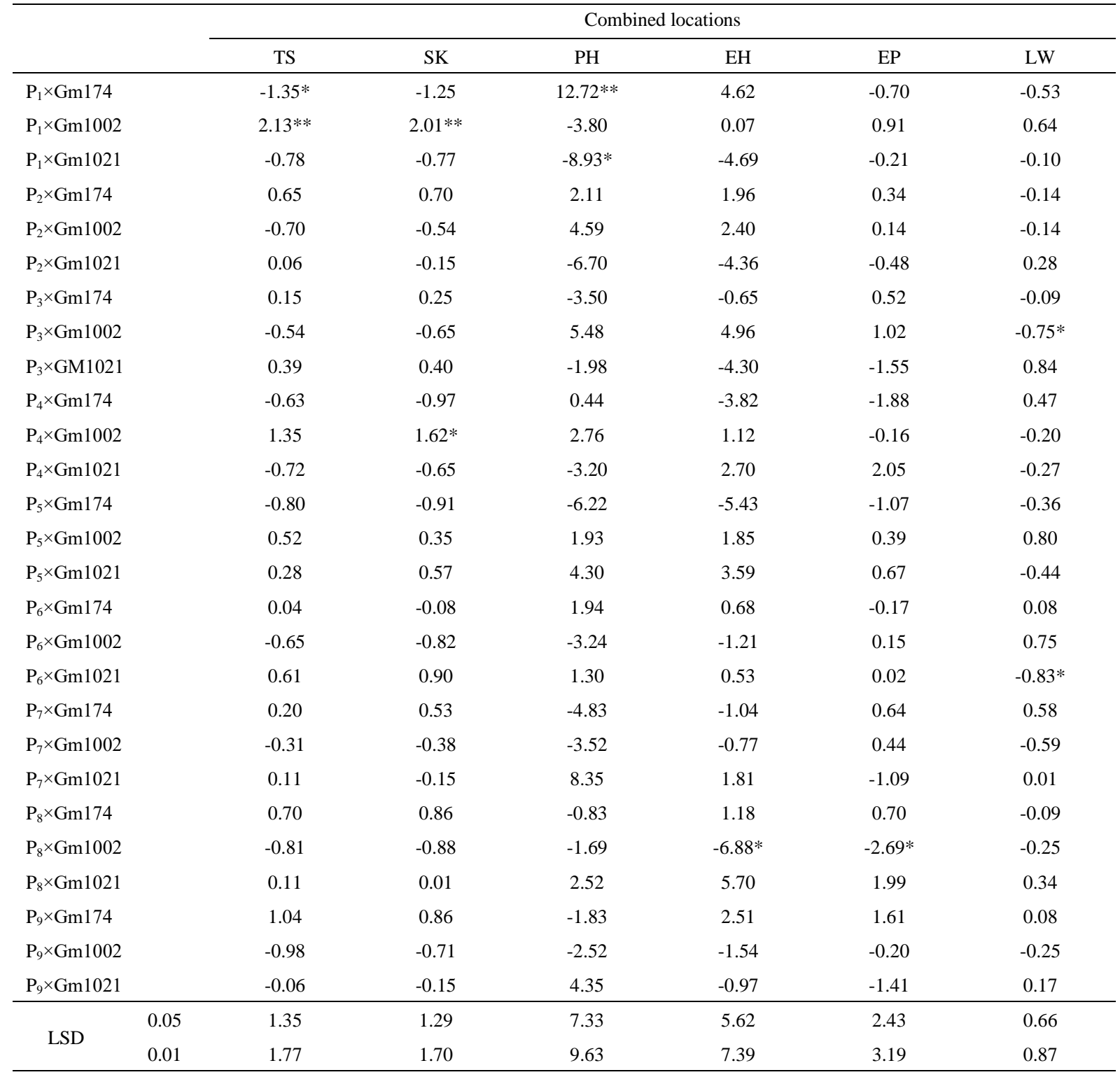

*,** significance at 0.05 and 0.01 level of probability, respectively 
Estimates of SCA effects of 27 yellow single crosses of maize at their combined data over locations for ear length, ear diameter, no. of rows per ear, no. of kernels per row, 100-kernel weight and grain yield in growing season 2016

\begin{tabular}{|c|c|c|c|c|c|c|c|}
\hline & & \multicolumn{6}{|c|}{ Combined locations } \\
\hline & & EL & ED & $\mathrm{RN}$ & $\mathrm{KN}$ & KW & GY \\
\hline $\mathrm{P}_{1} \times \mathrm{Gm} 174$ & & $1.55^{*}$ & 0.31 & $0.82 *$ & 3.08 & $3.78 *$ & $5.16^{* *}$ \\
\hline $\mathrm{P}_{1} \times \mathrm{Gm} 1002$ & & -1.04 & -0.31 & -0.46 & -3.09 & -1.33 & -0.99 \\
\hline $\mathrm{P}_{1} \times \mathrm{Gm} 1021$ & & -0.51 & 0.00 & -0.36 & 0.01 & -2.46 & -4.17 \\
\hline $\mathrm{P}_{2} \times \mathrm{Gm} 174$ & & -0.01 & -0.07 & 0.15 & -0.09 & 0.06 & 1.76 \\
\hline $\mathrm{P}_{2} \times \mathrm{Gm} 1002$ & & $2.07 * *$ & 0.30 & -0.29 & 1.41 & 1.45 & $2.81^{*}$ \\
\hline $\mathrm{P}_{2} \times \mathrm{Gm} 1021$ & & -2.06 & -0.22 & 0.14 & -1.33 & -1.51 & -4.58 \\
\hline $\mathrm{P}_{3} \times \mathrm{Gm} 174$ & & -0.78 & -0.13 & -0.29 & -1.42 & 1.06 & -0.97 \\
\hline $\mathrm{P}_{3} \times \mathrm{Gm} 1002$ & & $1.79 * *$ & 0.24 & 0.43 & 1.25 & -0.05 & 0.92 \\
\hline $\mathrm{P}_{3} \times \mathrm{GM} 1021$ & & -1.01 & -0.11 & -0.14 & 0.17 & -1.01 & 0.05 \\
\hline $\mathrm{P}_{4} \times \mathrm{Gm} 174$ & & $2.10 * *$ & 0.09 & 0.43 & 0.86 & 0.06 & 1.77 \\
\hline $\mathrm{P}_{4} \times \mathrm{Gm} 1002$ & & -1.82 & -0.20 & -0.68 & -1.98 & -3.72 & -0.91 \\
\hline $\mathrm{P}_{4} \times \mathrm{Gm} 1021$ & & -0.28 & 0.11 & 0.25 & 1.12 & $3.65 *$ & -0.86 \\
\hline $\mathrm{P}_{5} \times \mathrm{Gm} 174$ & & -0.23 & -0.19 & -0.85 & 0.47 & -1.22 & -2.27 \\
\hline $\mathrm{P}_{5} \times \mathrm{Gm} 1002$ & & -1.32 & 0.19 & $1.21^{* *}$ & -1.53 & -1.16 & -1.70 \\
\hline $\mathrm{P}_{5} \times \mathrm{Gm} 1021$ & & $1.55^{* *}$ & 0.00 & -0.36 & 1.06 & 2.38 & $3.97 * *$ \\
\hline $\mathrm{P}_{6} \times \mathrm{Gm} 174$ & & 0.72 & 0.04 & 0.38 & -1.53 & -0.83 & 0.25 \\
\hline $\mathrm{P}_{6} \times \mathrm{Gm} 1002$ & & -1.88 & -0.09 & -0.57 & 0.14 & -0.60 & -3.00 \\
\hline $\mathrm{P}_{6} \times \mathrm{Gm} 1021$ & & 1.16 & 0.06 & 0.19 & 1.40 & 1.43 & $2.75^{*}$ \\
\hline $\mathrm{P}_{7} \times \mathrm{Gm} 174$ & & -1.51 & -0.02 & -0.51 & -1.14 & -2.60 & -2.34 \\
\hline $\mathrm{P}_{7} \times \mathrm{Gm} 1002$ & & -0.27 & 0.02 & 0.71 & 0.52 & 2.12 & -0.96 \\
\hline $\mathrm{P}_{7} \times \mathrm{Gm} 1021$ & & $1.77 * *$ & 0.00 & -0.20 & 0.62 & 0.49 & $3.30^{* *}$ \\
\hline $\mathrm{P}_{8} \times \mathrm{Gm} 174$ & & -1.40 & 0.09 & 0.10 & -0.42 & 0.12 & -1.03 \\
\hline $\mathrm{P}_{8} \times \mathrm{Gm} 1002$ & & $1.01 *$ & -0.04 & -0.35 & -0.25 & 0.34 & 1.14 \\
\hline $\mathrm{P}_{8} \times \mathrm{Gm} 1021$ & & 0.38 & -0.06 & 0.25 & 0.67 & -0.46 & -0.11 \\
\hline $\mathrm{P}_{9} \times \mathrm{Gm} 174$ & & -0.45 & -0.13 & -0.23 & 0.19 & -0.44 & -2.34 \\
\hline $\mathrm{P}_{9} \times \mathrm{Gm} 1002$ & & 1.46 & -0.09 & -0.01 & 3.52 & 2.95 & $2.69 *$ \\
\hline $\mathrm{P}_{9} \times \mathrm{Gm} 1021$ & & -1.01 & 0.22 & 0.25 & -3.72 & -2.51 & -0.35 \\
\hline \multirow{2}{*}{ LSD } & 0.05 & 1.50 & 0.33 & 0.82 & 3.56 & 3.27 & 2.15 \\
\hline & 0.01 & 1.98 & 0.43 & 1.08 & 4.68 & 4.30 & 2.83 \\
\hline
\end{tabular}

*,** significance at 0.05 and 0.01 level of probability, respectively

\section{Superiority percentages}

It's to be noted that, negative values would be desired for no. of days to $50 \%$ tasseling and silking, plant height and ear height, ear position and disease infection; while the positive values would be favored for the other studied traits. Results given in Tables $(8$ 10) revealed that 27 cross combinations manifested negative and highly significant superiority percentages over than check varieties (SC162 and SC168) in combined data for days to $50 \%$ tasseling and silking date, ear height and ear position. For plant height, out of 27 crosses, 20 crosses had negative and highly significant superiority percentages over than check varieties (SC162 and SC168). For ear length, 7 crosses had positive and highly significant superiority percentages over than check varieties (SC162 and SC168). For ear diameter, 7 crosses had positive and highly significant superiority percentages over than check variety SC162 and 10 crosses had positive and highly significant superiority percentages over than check variety crosses SC168. For number of rows per ear, 7 and 12 crosses had positive and highly significant superiority percentages over than check varieties SC162 and SC168 respectively. For 100-kernel weight 7 crosses had positive and highly significant superiority percentages over than check variety SC162 and two crosses had positive and highly significant superiority percentages over than check variety SC168. For grain yield, 13 crosses had positive and highly significant superiority percentages over than check variety SC162 
and 14 crosses had positive and highly significant superiority percentages over than check variety cross SC168. Crosses $\mathrm{P}_{1} \times \mathrm{Gm} 174, \mathrm{P}_{2} \times \mathrm{Gm} 1002, \mathrm{P}_{5} \times \mathrm{Gm} 1021$, $\mathrm{P}_{6} \times \mathrm{Gm} 174, \mathrm{P}_{6} \times \mathrm{Gm} 1021, \mathrm{P}_{7} \times \mathrm{Gm} 1021, \mathrm{P}_{8} \times \mathrm{Gm1002}$, $\mathrm{P}_{9} \times \mathrm{Gm} 1021$ showed that best combinations manifested and significant superiority percentages over than check varieties for most studied traits and grain yield. These results agree with those obtained by Mosa, 2003; ELShenawy, 2005; Motawei, 2005; Barakat and Osman
2008; Sultan et al., 2010; EL-Hosary and Elgammaal, 2013; EL-Gazzar, et al., 2013; Osman, 2014; Aslam et al., 2017 and Shushay et al., 2017.

Based on the overall performance of the hybrids and parental lines, some of the lines could be used as parents of hybrids of maize with high grain yield potential. Thus, these crosses could be commercially exploited after critical evaluation for its superiority in performance as maize hybrids.

Table 8

Superiority percentages of the twenty seven $\mathrm{F}_{1}$ crosses relative to the two checks SC 162 and SC 168 for days to $50 \%$ tasseling, days to $50 \%$ silking, plant and ear height in their combined data over locations during in growing season 2016

\begin{tabular}{|c|c|c|c|c|c|c|c|c|c|}
\hline & & \multicolumn{8}{|c|}{ Combined locations } \\
\hline & & \multicolumn{2}{|c|}{ TS } & \multicolumn{2}{|c|}{ SK } & \multicolumn{2}{|c|}{$\mathrm{PH}$} & \multicolumn{2}{|c|}{$\mathrm{EH}$} \\
\hline & & SC162 & SC168 & SC162 & SC168 & SC162 & SC168 & SC162 & SC168 \\
\hline \multicolumn{2}{|c|}{$\mathrm{P}_{1} \times \mathrm{Gm} 174$} & $-13.78 * *$ & $-12.15^{* *}$ & $-13.94 * *$ & $-13.03 * *$ & -7.48 & -7.29 & $-31.33 * *$ & $-29.61 * *$ \\
\hline \multicolumn{2}{|c|}{$\mathrm{P}_{1} \times \mathrm{Gm} 1002$} & $-4.86^{* *}$ & $-4.86^{* *}$ & $-7.11^{* *}$ & $-6.12 * *$ & $-13.61 *$ & $-13.43^{*}$ & $-34.36 * *$ & $-32.72 * *$ \\
\hline \multicolumn{2}{|c|}{$\mathrm{P}_{1} \times \mathrm{Gm} 1021$} & $-13.26^{* *}$ & $-11.62 * *$ & $-13.42^{* *}$ & $-12.50^{* *}$ & $-17.06^{*}$ & $-16.89^{* *}$ & $-38.63 * *$ & $-37.09 * *$ \\
\hline \multicolumn{2}{|c|}{$\mathrm{P}_{2} \times \mathrm{Gm} 174$} & $-12.99 * *$ & $-11.34 * *$ & $-12.88^{* *}$ & $-11.96^{* *}$ & -12.59 & -12.41 & $-34.36 * *$ & $-32.72 * *$ \\
\hline \multicolumn{2}{|c|}{$\mathrm{P}_{2} \times \mathrm{Gm} 1002$} & $-13.53^{* *}$ & $-11.89 * *$ & $-13.15^{* *}$ & $-12.23^{* *}$ & -11.44 & -11.26 & $-34.55^{* *}$ & $-32.91 * *$ \\
\hline \multicolumn{2}{|c|}{$\mathrm{P}_{2} \times \mathrm{Gm} 1021$} & $-14.32 * *$ & $-12.71 * *$ & $-14.46^{* *}$ & $-13.56^{* *}$ & $-17.25^{* *}$ & $-17.08^{* *} *$ & $-39.95 * *$ & $-38.45 * *$ \\
\hline \multicolumn{2}{|c|}{$\mathrm{P}_{3} \times \mathrm{Gm} 174$} & $-12.99 * *$ & $-11.34 * *$ & $-12.88^{* *}$ & $-11.96^{* *}$ & $-14.63 *$ & $-14.46^{*}$ & $-34.46^{* *}$ & $-32.82 * *$ \\
\hline \multicolumn{2}{|c|}{$\mathrm{P}_{3} \times \mathrm{Gm} 1002$} & $-12.46^{* *}$ & $-10.81^{* *}$ & $-12.63^{* *}$ & $-11.71^{* *}$ & -10.99 & -10.81 & $-31.71 * *$ & $-30.00 * *$ \\
\hline \multicolumn{2}{|c|}{$\mathrm{P}_{3} \times \mathrm{GM} 1021$} & $-12.99 * *$ & $-11.34 * *$ & $-12.88 * *$ & $-11.96^{* *}$ & $-15.34 *$ & $-15.16^{*}$ & $-38.53 * *$ & $-36.99 * *$ \\
\hline \multicolumn{2}{|c|}{$\mathrm{P}_{4} \times \mathrm{Gm} 174$} & $-13.78^{* *}$ & $-12.15^{* *}$ & $-14.46^{* *}$ & $-13.56^{* *}$ & $-15.34 *$ & $-15.16^{*}$ & $-39.10 * *$ & $-37.57 * *$ \\
\hline \multicolumn{2}{|c|}{$\mathrm{P}_{4} \times \mathrm{Gm} 1002$} & $-9.01 * *$ & $-7.29 * *$ & $-8.68 * *$ & $-7.72 * *$ & $-14.25^{*}$ & $-14.07^{*}$ & $-36.73 * *$ & $-35.15^{* *}$ \\
\hline \multicolumn{2}{|c|}{$\mathrm{P}_{4} \times \mathrm{Gm} 1021$} & $-14.32 * *$ & $-12.71 * *$ & $-14.21^{* *}$ & $-13.30 * *$ & $-18.02 * *$ & $-17.85^{* *}$ & $-37.39 * *$ & $-35.82 * *$ \\
\hline \multicolumn{2}{|c|}{$\mathrm{P}_{5} \times \mathrm{Gm} 174$} & $-14.85^{* *}$ & $-13.24^{* *}$ & $-15.25^{* *}$ & $-14.35^{* *}$ & $-15.34 *$ & $-15.16^{*}$ & $-37.11 * *$ & $-35.53^{* *}$ \\
\hline \multicolumn{2}{|c|}{$\mathrm{P}_{5} \times \mathrm{Gm} 1002$} & $-11.14^{* *}$ & $-9.46 * *$ & $-11.57 * *$ & $-10.64 * *$ & -12.01 & -11.83 & $-33.41 * *$ & $-31.75^{* *}$ \\
\hline \multicolumn{2}{|c|}{$\mathrm{P}_{5} \times \mathrm{Gm} 1021$} & $-13.53^{* *}$ & $-11.89 * *$ & $-13.15^{* *}$ & $-12.23 * *$ & -12.59 & -12.41 & $-33.98 * *$ & $-32.33^{* *}$ \\
\hline \multicolumn{2}{|c|}{$\mathrm{P}_{6} \times \mathrm{Gm} 174$} & $-13.53 * *$ & $-11.89 * *$ & $-13.42^{* *}$ & $-12.50^{* *}$ & $-14.06^{*}$ & $-13.88 *$ & $-37.02 * *$ & $-35.44 * *$ \\
\hline \multicolumn{2}{|c|}{$\mathrm{P}_{6} \times \mathrm{Gm} 1002$} & $-12.99 * *$ & $-11.34 * *$ & $-12.88^{* *}$ & $-11.96^{* *}$ & $-15.85^{*}$ & $-15.67^{*}$ & $-38.53 * *$ & $-36.99 * *$ \\
\hline \multicolumn{2}{|c|}{$\mathrm{P}_{6} \times \mathrm{Gm} 1021$} & $-12.99 * *$ & $-11.34 * *$ & $-12.10^{* *}$ & $-11.16^{* *}$ & $-15.59 *$ & $-15.42 *$ & $-39.10 * *$ & $-37.57 * *$ \\
\hline \multicolumn{2}{|c|}{$\mathrm{P}_{7} \times \mathrm{Gm} 174$} & $-13.53^{* *}$ & $-11.89 * *$ & $-12.63^{* *}$ & $-11.71^{* *}$ & $-14.76^{*}$ & $-14.59 *$ & $-34.65 * *$ & $-33.01 * *$ \\
\hline \multicolumn{2}{|c|}{$\mathrm{P}_{7} \times \mathrm{Gm} 1002$} & $-12.73 * *$ & $-11.08 * *$ & $-12.36^{* *}$ & $-11.43^{* *}$ & $-14.06^{*}$ & $-13.88^{*}$ & $-34.93 * *$ & $-33.30 * *$ \\
\hline \multicolumn{2}{|c|}{$\mathrm{P}_{7} \times \mathrm{Gm} 1021$} & $-14.05^{* *}$ & $-12.43^{* *}$ & $-13.94 * *$ & $-13.03 * *$ & -10.99 & -10.81 & $-35.03 * *$ & $-33.40 * *$ \\
\hline \multicolumn{2}{|c|}{$\mathrm{P}_{8} \times \mathrm{Gm} 174$} & $-12.73^{* *}$ & $-11.08 * *$ & $-12.63^{* *}$ & $-11.71^{* *}$ & $-14.38 *$ & $-14.20 *$ & $-35.50 * *$ & $-33.88 * *$ \\
\hline \multicolumn{2}{|c|}{$\mathrm{P}_{8} \times \mathrm{Gm} 1002$} & $-13.53 * *$ & $-11.89 * *$ & $-13.67 * *$ & $-12.76^{* *}$ & $-14.50 *$ & $-14.33^{*}$ & $-40.52 * *$ & $-39.03 * *$ \\
\hline $\mathrm{P}_{8} \times \mathrm{Gm}$ & & $-14.05^{* *}$ & $-12.43^{* *}$ & $-14.21^{* *}$ & $-13.30^{* *}$ & $-14.38 *$ & $-14.20 *$ & $-34.93 * *$ & $-33.30 * *$ \\
\hline $\mathrm{P}_{9} \times \mathrm{Gm}$ & & $-12.73^{* *}$ & $-11.08^{* *}$ & $-13.15^{* *}$ & $-12.23^{* *}$ & $-16.29 *$ & $-16.12 *$ & $-35.31 * *$ & $-33.69 * *$ \\
\hline $\mathrm{P}_{9} \times \mathrm{Gm}$ & & $-14.32 * *$ & $-12.71 * *$ & $-13.94^{* *}$ & $-13.03 * *$ & $-16.36^{*}$ & $-16.19 *$ & $-38.06 * *$ & $-36.51 * *$ \\
\hline $\mathrm{P}_{9} \times \mathrm{Gm}$ & & $-12.29 * *$ & $-13.24 * *$ & $-15.00^{* *}$ & $-14.11^{* *}$ & $-15.21 *$ & $-15.03^{*}$ & $-39.29 * *$ & $-37.77^{* *}$ \\
\hline & 0.05 & & & & & & & & \\
\hline & 0.01 & & & & & & & & \\
\hline
\end{tabular}

*** significance at 0.05 and 0.01 level of probability, respectively 
Table 9

Superiority percentages of the twenty seven $F_{1}$ crosses relative to the two checks SC 162 and SC 168 for ear position, late wilt disease, ear length and ear diameter in their combined data over locations during in growing season 2016

\begin{tabular}{|c|c|c|c|c|c|c|c|c|c|}
\hline & & \multicolumn{8}{|c|}{ Combined locations } \\
\hline & & \multicolumn{2}{|c|}{$\mathrm{EP}$} & \multicolumn{2}{|c|}{ LW } & \multicolumn{2}{|c|}{ EL } & \multicolumn{2}{|c|}{ ED } \\
\hline & & $\mathrm{SC} 162$ & SC168 & $\mathrm{SC} 162$ & $\mathrm{SC} 168$ & $\mathrm{SC} 162$ & SC168 & $\mathrm{SC} 162$ & $\mathrm{SC} 168$ \\
\hline \multicolumn{2}{|c|}{$\mathrm{P}_{1} \times \mathrm{Gm} 174$} & $-25.81^{* *}$ & $-24.08 * *$ & -0.20 & -0.30 & $5.05^{* *}$ & $5.45^{* *}$ & $5.26^{* *}$ & $5.88 * *$ \\
\hline \multicolumn{2}{|c|}{$\mathrm{P}_{1} \times \mathrm{Gm} 1002$} & $-24.16^{* *}$ & $-22.39 * *$ & $-2.00 * *$ & $-2.20 * *$ & -15.53 & -15.20 & -13.84 & -13.33 \\
\hline \multicolumn{2}{|c|}{$\mathrm{P}_{1} \times \mathrm{Gm} 1021$} & $-26.33^{* *}$ & $-24.61 * *$ & -0.70 & $-1.80 * *$ & -4.66 & -4.30 & -2.14 & -1.57 \\
\hline \multicolumn{2}{|c|}{$\mathrm{P}_{2} \times \mathrm{Gm} 174$} & $-25.07 * *$ & $-23.33 * *$ & 0.00 & 0.00 & 1.59 & 1.98 & -0.58 & 0.00 \\
\hline \multicolumn{2}{|c|}{$\mathrm{P}_{2} \times \mathrm{Gm} 1002$} & $-26.10^{* *}$ & $-24.37 * *$ & -0.70 & -0.80 & $3.70^{* *}$ & $4.10^{* *}$ & $1.95^{* *}$ & $2.55^{* *}$ \\
\hline \multicolumn{2}{|c|}{$\mathrm{P}_{2} \times \mathrm{Gm} 1021$} & $-27.53 * *$ & $-25.84 * *$ & -0.50 & -1.00 & -9.23 & -8.88 & -3.51 & -2.94 \\
\hline \multicolumn{2}{|c|}{$\mathrm{P}_{3} \times \mathrm{Gm} 174$} & $-23.30^{* *}$ & $-21.51^{* *}$ & -0.70 & -1.00 & -9.95 & -9.60 & -4.48 & -3.92 \\
\hline \multicolumn{2}{|c|}{$\mathrm{P}_{3} \times \mathrm{Gm} 1002$} & $-23.30 * *$ & $-21.51^{* *}$ & -0.70 & -0.70 & -5.91 & -5.55 & 0.00 & $0.59 *$ \\
\hline \multicolumn{2}{|c|}{$\mathrm{P}_{3} \times \mathrm{GM} 1021$} & $-27.61^{* *}$ & $-25.92 * *$ & $-1.70 * *$ & $-2.20 * *$ & -10.91 & -10.57 & -3.51 & -2.94 \\
\hline \multicolumn{2}{|c|}{$\mathrm{P}_{4} \times \mathrm{Gm} 174$} & $-28.14^{* *}$ & $-26.46^{* *}$ & 0.00 & $-1.30 *$ & $4.95^{* *}$ & $5.36^{* *}$ & $1.36^{* *}$ & $1.96^{* *}$ \\
\hline \multicolumn{2}{|c|}{$\mathrm{P}_{4} \times \mathrm{Gm} 1002$} & $-26.32 * *$ & $-24.60 * *$ & -0.80 & $-1.30^{*}$ & -22.26 & -21.96 & -10.33 & -9.80 \\
\hline \multicolumn{2}{|c|}{$\mathrm{P}_{4} \times \mathrm{Gm} 1021$} & $-23.56^{* *}$ & $-21.78 * *$ & -0.20 & -0.20 & $-6.73 * *$ & -6.37 & -2.53 & -1.96 \\
\hline \multicolumn{2}{|c|}{$\mathrm{P}_{5} \times \mathrm{Gm} 174$} & $-25.89 * *$ & $-24.16^{* *}$ & -0.70 & $-1.50 *$ & -4.95 & -4.58 & -3.90 & -3.33 \\
\hline \multicolumn{2}{|c|}{$\mathrm{P}_{5} \times \mathrm{Gm} 1002$} & $-24.45^{* *}$ & $-22.69 * *$ & $-2.50 * *$ & $-2.80 * *$ & -16.97 & -16.65 & -1.17 & -0.59 \\
\hline \multicolumn{2}{|c|}{$\mathrm{P}_{5} \times \mathrm{Gm} 1021$} & $-24.56^{* *}$ & $-22.79 * *$ & -0.70 & -0.80 & $4.33^{* *}$ & $4.73 * *$ & 0.00 & $0.59 *$ \\
\hline \multicolumn{2}{|c|}{$\mathrm{P}_{6} \times \mathrm{Gm} 174$} & $-26.91 * *$ & $-25.20 * *$ & -0.80 & -1.00 & $6.88^{* * *}$ & $7.29 * *$ & $3.31^{* *}$ & $3.92 * *$ \\
\hline \multicolumn{2}{|c|}{$\mathrm{P}_{6} \times \mathrm{Gm} 1002$} & $-27.18^{* *}$ & $-25.48 * *$ & $-2.20 * *$ & $-2.20 * *$ & -13.46 & -13.13 & -2.53 & -1.96 \\
\hline \multicolumn{2}{|c|}{$\mathrm{P}_{6} \times \mathrm{Gm} 1021$} & $-27.89 * *$ & $-26.20 * *$ & 0.00 & 0.00 & $9.13^{* *}$ & $9.56^{* *}$ & $3.31 * *$ & $3.92 * *$ \\
\hline \multicolumn{2}{|c|}{$\mathrm{P}_{7} \times \mathrm{Gm} 174$} & $-23.33^{* *}$ & $-21.54 * *$ & -0.70 & -0.70 & -8.80 & -8.45 & -3.90 & -3.33 \\
\hline \multicolumn{2}{|c|}{$\mathrm{P}_{7} \times \mathrm{Gm} 1002$} & $-24.35^{* *}$ & $-22.58 * *$ & -0.20 & -0.30 & -10.91 & -10.57 & -4.48 & -3.92 \\
\hline \multicolumn{2}{|c|}{$\mathrm{P}_{7} \times \mathrm{Gm} 1021$} & $-27.13^{* *}$ & $-25.43^{* *}$ & -0.20 & -0.30 & $7.69 * *$ & $8.11^{* *}$ & 0.39 & $0.98^{* *}$ \\
\hline \multicolumn{2}{|c|}{$\mathrm{P}_{8} \times \mathrm{Gm} 174$} & $-24.79 * *$ & $-23.04 * *$ & -0.20 & -0.20 & -9.13 & -8.78 & $3.31 * *$ & $3.92 * *$ \\
\hline \multicolumn{2}{|c|}{$\mathrm{P}_{8} \times \mathrm{Gm} 1002$} & $-30.52 * *$ & $-28.90 * *$ & -0.70 & $-1.20^{*}$ & -4.95 & -4.58 & -5.85 & -5.29 \\
\hline $\mathrm{P}_{8} \times \mathrm{Gm}$ & & $-24.13^{* *}$ & $-22.36^{* *}$ & -0.70 & -0.50 & 0.34 & 0.72 & -0.58 & 0.00 \\
\hline $\mathrm{P}_{9} \times \mathrm{Gm}$ & & $-22.84 * *$ & $-21.04 * *$ & -0.30 & -0.30 & -6.15 & -5.79 & -4.48 & -3.92 \\
\hline $\mathrm{P}_{9} \times \mathrm{Gm}$ & & $-26.24 * *$ & $-24.52 * *$ & -0.70 & -1.00 & -3.99 & -3.62 & -8.38 & -7.84 \\
\hline $\mathrm{P}_{9} \times \mathrm{Gm}$ & & $-28.55^{* *}$ & $-26.89^{* *}$ & -0.50 & $-1.20^{*}$ & -8.08 & -7.72 & $0.78^{* *}$ & $1.37 * *$ \\
\hline I SD & 0.05 & & & & & & & & \\
\hline & 0.01 & & & & & & & & \\
\hline
\end{tabular}

*,** significance at 0.05 and 0.01 level of probability, respectively 
Superiority percentages of the twenty seven $F_{1}$ crosses relative to the two checks SC 162 and SC 168 for no. of rows per ear, no. of kernels per rows, 100-kernels weight and grain yield in their combined data over locations during in growing season 2016

\begin{tabular}{|c|c|c|c|c|c|c|c|c|c|}
\hline & & \multicolumn{8}{|c|}{ Combined locations } \\
\hline & & \multicolumn{2}{|c|}{$\mathrm{RN}$} & \multicolumn{2}{|c|}{$\mathrm{KN}$} & \multicolumn{2}{|c|}{$\mathrm{KW}$} & \multicolumn{2}{|c|}{ GY } \\
\hline & & SC162 & SC168 & SC162 & $\mathrm{SC} 168$ & SC162 & SC168 & SC162 & SC168 \\
\hline $\mathrm{P}_{1} \times \mathrm{Gm}$ & & $4.02 * *$ & $5.20 * *$ & -1.06 & -3.93 & $9.27 * *$ & 3.58 & $22.75^{* *}$ & $23.76^{* *}$ \\
\hline $\mathrm{P}_{1} \times \mathrm{Gm}$ & & -4.22 & -3.13 & -18.88 & -21.23 & -11.32 & -15.94 & -2.97 & -2.97 \\
\hline $\mathrm{P}_{1} \times \mathrm{Gm}$ & & -2.44 & -1.33 & -8.84 & -11.48 & -6.22 & -11.10 & -4.16 & -3.38 \\
\hline $\mathrm{P}_{2} \times \mathrm{Gm}$ & & 0.66 & $1.80 *$ & -5.41 & -8.15 & $8.54 * *$ & 2.89 & $16.75^{* *}$ & $17.70 * *$ \\
\hline $\mathrm{P}_{2} \times \mathrm{Gm}$ & & -1.58 & -0.47 & -5.17 & -7.92 & 4.82 & -0.64 & $11.03^{* *}$ & $11.94 * *$ \\
\hline $\mathrm{P}_{2} \times \mathrm{Gm}$ & & $1.85^{*}$ & $3.00^{* *}$ & -8.54 & -11.19 & 5.21 & -0.27 & -1.50 & -0.70 \\
\hline $\mathrm{P}_{3} \times \mathrm{Gm}$ & & -7.71 & -6.67 & -6.58 & -9.29 & 0.29 & -4.93 & 0.14 & 0.96 \\
\hline $\mathrm{P}_{3} \times \mathrm{Gm}$ & & -2.44 & -1.33 & -4.37 & -7.15 & -10.02 & -14.71 & -3.15 & -2.36 \\
\hline $\mathrm{P}_{3} \times \mathrm{GM}$ & & -3.76 & -2.67 & -3.83 & -6.62 & -5.00 & -9.94 & 3.09 & $3.93^{*}$ \\
\hline $\mathrm{P}_{4} \times \mathrm{Gm}$ & & -1.32 & -0.20 & -5.71 & -8.45 & 2.99 & -2.37 & $9.18^{* *}$ & $10.07^{* *}$ \\
\hline $\mathrm{P}_{4} \times \mathrm{Gm}$ & & -8.17 & -7.13 & -16.53 & -18.95 & -13.66 & -18.16 & -7.33 & -6.58 \\
\hline $\mathrm{P}_{4} \times \mathrm{Gm}$ & & -0.99 & 0.13 & -6.58 & -9.29 & $13.30^{* *}$ & $7.40^{* *}$ & 1.62 & 2.45 \\
\hline $\mathrm{P}_{5} \times \mathrm{Gm}$ & & -4.22 & -3.13 & -4.14 & -6.92 & 4.03 & -1.38 & -9.79 & -9.05 \\
\hline $\mathrm{P}_{5} \times \mathrm{Gm}$ & & $9.89 * *$ & $11.13^{* * *}$ & -12.93 & -15.46 & -3.88 & -8.88 & -16.89 & -16.22 \\
\hline $\mathrm{P}_{5} \times \mathrm{Gm}$ & & 0.99 & $2.13 * *$ & -4.23 & -7.01 & $13.66^{* *}$ & $7.75^{* *}$ & $8.26 * *$ & $9.14 * *$ \\
\hline $\mathrm{P}_{6} \times \mathrm{Gm}$ & & $8.44 * *$ & $9.67 * *$ & -7.83 & -10.50 & 4.19 & -1.23 & $13.34 * *$ & $14.26^{* *}$ \\
\hline $\mathrm{P}_{6} \times \mathrm{Gm}$ & & $2.04 * *$ & $3.20 * *$ & -8.30 & -10.96 & -3.18 & -8.22 & -4.82 & -4.05 \\
\hline $\mathrm{P}_{6} \times \mathrm{Gm}$ & & $7.25 * *$ & $8.47 * *$ & -1.95 & -4.79 & $10.54 * *$ & 4.79 & $20.56^{* *}$ & $21.54^{* *}$ \\
\hline $\mathrm{P}_{7} \times \mathrm{Gm}$ & & -5.08 & -4.00 & -6.35 & -9.06 & -2.92 & -7.97 & -2.57 & -1.78 \\
\hline $\mathrm{P}_{7} \times \mathrm{Gm}$ & & $3.69 * *$ & $4.87 * *$ & -5.41 & -8.15 & 0.99 & -4.27 & -7.33 & -6.58 \\
\hline $\mathrm{P}_{7} \times \mathrm{Gm}$ & & -0.26 & 0.87 & -2.66 & -5.48 & 5.67 & 0.17 & $13.75^{* *}$ & $14.67 * *$ \\
\hline $\mathrm{P}_{8} \times \mathrm{Gm}$ & & 1.38 & $2.53 * *$ & -2.59 & -5.41 & $9.66 * *$ & 3.95 & $8.29 * *$ & $9.17 * *$ \\
\hline $\mathrm{P}_{8} \times \mathrm{Gm}$ & & -0.79 & 0.33 & -5.71 & -8.45 & 2.47 & -2.86 & $5.80 * *$ & $6.67 * *$ \\
\hline $\mathrm{P}_{8} \times \mathrm{Gm}$ & & $3.03 * *$ & $4.20 * *$ & -1.01 & -3.88 & $8.54 * *$ & 2.89 & $10.97^{* *}$ & $11.88^{* *}$ \\
\hline $\mathrm{P}_{9} \times \mathrm{Gm}$ & & -4.28 & -3.20 & -8.06 & -10.73 & 3.15 & -2.22 & -1.10 & -0.29 \\
\hline $\mathrm{P}_{9} \times \mathrm{Gm}$ & & -2.11 & -1.00 & -4.23 & -7.01 & 4.29 & -1.13 & $4.65^{*}$ & $5.50 * *$ \\
\hline $\mathrm{P}_{9} \times \mathrm{Gm}$ & & $1.65^{*}$ & $2.80^{* *}$ & -2.94 & -5.75 & -1.30 & -6.44 & $4.65^{*}$ & $5.50 * *$ \\
\hline \multirow{2}{*}{ LSD } & 0.05 & \multicolumn{2}{|c|}{1.43} & \multicolumn{2}{|c|}{6.21} & \multicolumn{2}{|c|}{5.69} & \multicolumn{2}{|c|}{3.77} \\
\hline & 0.01 & \multicolumn{2}{|c|}{1.88} & \multicolumn{2}{|c|}{8.14} & \multicolumn{2}{|c|}{7.46} & \multicolumn{2}{|c|}{4.94} \\
\hline
\end{tabular}

**** significance at 0.05 and 0.01 level of probability, respectively

\section{CONCLUSION}

In conclusion, results were showed that significance and highly significance for most studied traits (days to $50 \%$ tasseling, days to $50 \%$ silking, plant and ear height, ear position, ear length, no. of kernels per row, 100 -kernel weight and Grain yield). Estimates variance were due to GCA and SCA and their ratio revealed predominantly non-additive gene effects for all studied traits. Lines with the best GCA effects were: $\mathrm{P}_{2}$ (line 11) and $P_{6}$ (line 21) for grain yield, for testers $G m 174$ and Gm1021 had significant GCA effects for grain yield. The hybrids $\mathrm{P}_{5} \times \mathrm{Gm} 1021, \mathrm{P}_{6} \times \mathrm{Gm} 1021$, $\mathrm{P}_{7} \times \mathrm{Gm} 1021, \mathrm{P}_{8} \times \mathrm{Gm} 1002, \mathrm{P}_{9} \times \mathrm{Gm} 1002$ had significant and negative SCA effects for grain yield. Crosses $\mathrm{P}_{1} \times \mathrm{Gm} 174, \mathrm{P}_{2} \times \mathrm{Gm} 1002, \mathrm{P}_{5} \times \mathrm{Gm} 1021, \quad \mathrm{P}_{6} \times \mathrm{Gm} 174$, $\mathrm{P}_{6} \times \mathrm{Gm} 1021, \mathrm{P}_{7} \times \mathrm{Gm} 1021, \mathrm{P}_{8} \times \mathrm{Gm} 1002, \mathrm{P}_{9} \times \mathrm{Gm} 1021$ were the best combinations manifested and significant superiority percentages over than check varieties (SC162 and SC168) for most studied traits. Therefore, these hybrids may be preferred for hybrid crop development. 


\section{REFERENCES}

Agrobase 21 (2001): Agronomix Software, Inc. 171 Waterloo Street Winnipeg, Manitoba, Canada R3N OS4, www. Agronomix.mb.ca.

Asefa, B.-Mohammed, H.-Zelleke, H. (2008): Combining ability of transitional high land maize inbred lines. East African J. Sci. 2, $19-24$.

Aslam, M.-Sohail, Q.-Maqbool, M. A.-Ahmad, S.-Shahzad, R. (2017): Combining Ability Analysis for yield traits in diallel crosses of maize. J. of Ani. \& Pl. Sci., 27(1): 136-143.

Atif, I. A.-Awadalla, A. A.-Mutasim, M. (2012): Combining Ability and Heterosis for Yield and Yield Components in Maize (Zea mays L.). Australian J. of Basic and Applied Sci. 6(10), 36-41.

Barakat, A. A.-Osman, M. M. A. (2008): Evaluation of some newly developed yellow maize inbred lines for combining ability in two locations. J. Agric. Sci Mansoura Univ., 33(7): 4667-4679.

Ceyhan, E.-Avci, M.-Karada, S. (2008): Line X tester analysis in pea (Pisum sativum L.) Identification of superior parents for seed yield and its components. African J.of Biotec. 7(16), 2810-2817.

El-Shenawy, A. A. (2005): Combining ability of prolific and nonprolific maize inbred lines in their diallel crosses for yield and other traits. J. Agric. Res. Tanta Univ., 31(1): 16-31.

El-Shenawy, A. A.-Mosa, H. E.-Motawei, A. A. (2009): Combining Ability of crosses and Stability Parameters of their Single Crosses. J. Agri. Res. 35(4): 940-953.

EL-Gazzar, I. A. I.-EL-Ghonemy, M. A.-Mousa, S. Th. (2013) Evaluation of new inbred lines of white maize via line $\mathrm{x}$ tester analysis over three locations. J. Plant Production, Mansoura Univ., Vol. 4 (6): 897-906, 2013.

EL-Hosary, A. A. A.-Elgammaal, A. A. (2013): Utilization of line x tester model for evaluating the combining ability of some new white maize inbred lines. Egypt. J. Plant Breed. 17 (1):79-72.

Fan, X. M.-Zhang, Y. M.-Yao, W. H.-Chen. H. M.-Tan, J.-Han, X. L.-Luo, L. M.-Kang, M. S. (2009): Classifying maize inbred lines into heterotic groups using a factorial mating design. Agron. J., 101: 106-112.

FAO Statistics (2016): www.fao.org.

Kamara, M. M.- El-Degwy, I. S.-Koyama, H. (2014): Estimation of combining ability of some maize inbred lines using line $\times$ tester mating design under two nitrogen levels. AJCS 8(9):1336-1342.

Kempthorne, O. (1957): An introduction to genetic statics. Johan wily and sons, Inc. New York, USA.468-473.

Liu, W.-Tollenaar, M. (2009): Physiological mechanisms underlying heterosis for shade tolerance in maize. Crop Sci. 49:1817-1826.

Moosavi, S. G.-Seghatoleslami, M. J.-Moazeni, A. (2012): Effect of planting date and plant density on morphological traits, LAI and forage corn (Sc. 370) yield in second cultivation. Azad University, Iran Inter. Res. J. of Appl. and Basic Sci. 3 (1): 57 63.
Mosa, H. E. (2003): Heterosis and combining ability in maize (Zea mays L.). Minufiya J. Agric. Res., 28(5): 1375-1386.

Motawei, A. A. (2005): Combining ability and heterotic effect of nine maize inbred lines via diallel cross analysis. Minufiya J. Agric. Res. 30 (1): 197-214.

Osman, M. M. A. (2014): A diallel analysis among seven newly yellow maize inbred lines for grain yield and other agronomic traits. Egypt. J. of Appl. Sci.; 29 (1): 1-11.

Osman, M. M. A.-Ibrahim, M. H. A. (2007): A study on combining ability of new lines using line $\mathrm{x}$ tester analysis. J. Agric. Sci. Mansoura Univ., 32(2): 815-830.

Parmar, H. P. (2007): Combining ability and stability analysis in forage maize (Zea mays L.). Ph. D. Thesis B.A. College of Agric., Gujarat Agric. Univ., Anand campus, India.

Rahman, H.-Arifuddin, Z.-Shah, S. M.-Shah, A.Iqbal, M.-Khalil, I. H. (2010): Evaluation of maize in test cross combinations; flowering and morphological traits. Pakistan J. Botany 42(3), 1619-1627.

Rakesh Kumar-Mohinder, S.-Narwal, M. S. (2006): Combining ability analysis for grain yield and its contributing traits in maize (Zea mays L.). National J. of Plant Improv., 8 (1): 62-66.

Sharief, A. E.-El-Kalla, S. E.-Gado, H. E.-Yousef, H. (2009): Heterosis in yellow maize. Australian J. Crop Sci. 3, 146-154.

Sharma, S.-Narwal, M. S.-Kumar, R.-Dass, S. (2004): Line x tester analysis in maize (Zea mays L.). Forage Research 30, 28-30.

Shushay W. Abrha-Habtamu Z. Zeleke-Dagne W. Gissa (2017). Line $\mathrm{x}$ tester analysis of maize inbred lines for grain yield and yield related traits. Asian J. of Plant Scie. and Res., 3(5):12-19.

Singh, P. K.-Roy, A. K. (2007). Diallel analysis of inbred lines in maize (Zea mays L.). International J. of Agric. Sci. 3(1): 213216.

Steel, R. G.-Torrie, J. H. (1980): Principle and procedures of Statistics. Me. Grow Hill Book. Tne., New York, USA.

Sultan, M. S.-Abdel-Monaem, M. A.-Haffez, S. H. (2010): combining ability and heterosis estimates for yield, yield components and quality traits in maize under two plant densities. J. Agric. Sic., Mansoura Univ., 1(10):1419-1430.

Welcker, C. C.-Andréau, B. C.-Parentoni, S. N.-Bernal, J.-Horst, W. J. (2005): Heterosis and combining ability for maize adaptation to tropical acid soils. Crop Sci., 45: 2405-2413. 